\title{
Analysis of spectral broadening dominated by cascaded stimulated Raman scattering in optical fibers
}

\author{
D. Torres-González, J. M. Sierra-Hernández, D. Jáuregui-Vázquez, \\ J. R. Reyes-Ayona, M. Avazpour, J. M. Estudillo-Ayala and R. Rojas-Laguna \\ División de Ingenierías Campus Irapuato-Salamanca, Universidad de Guanajuato, \\ Carretera Salamanca-Valle de Santiago, km. 3.5 + 1.8. Comunidad de Palo Blanco, Salamanca, Guanajuato, México. \\ e-mail: rlaguna@ugto.mx \\ J. C. García-Hernández \\ Catedras, Consejo Nacional de Ciencia y Tecnología, Dirección Adjunta de Desarrollo Científico, \\ Avenida Insurgentes Sur 1582, Crédito Constructor, Ciudad de México, México.
}

Received 14 November 2020; accepted 23 December 2020

In this work, the experimental study of spectral broadening due to stimulated Raman scattering with respect to the variation of the input power of a pulsed laser, as well as a $4 \mathrm{~m}$ length high-numerical aperture fiber with 100 and $500 \mathrm{~m}$ of non-zero dispersion-shifted fiber is reported. The results showed an extreme broadening of the spectrum, known as supercontinuum generation, originated by self-phase modulation, four-wave mixing, and dominated by cascaded stimulated Raman scattering. Supercontinuum spectrum achieved by total width of $850 \mathrm{~nm}$ and a remarkable flatness in the range of 1500 to $1600 \mathrm{~nm}$. Furthermore, the output spectrum showed a total of five Stokes waves. Numerical results are also presented.

Keywords: Optical fibers; stimulated Raman scattering; supercontinuum generation.

\section{Introduction}

The study of the nonlinear effect known as stimulated Raman scattering (SRS) in optical fibers is of great importance due to the solutions it brings, mainly in telecommunications, since it is a versatile technique for light amplification whose gain is not fixed to a frequency bandwidth range, contrary to the gain spectrum of rare-earth-doped fibers. However, care must be taken in a long-haul multichannel communication system if one of the propagating signals has a high power enough to trigger the Raman effect.

Raman effect was first observed and described by C. V. Raman in 1928 [1,2]. The study of SRS in optical fibers began in early the 1970 decade, where Raman gain was quickly exploited to the development of amplifiers and oscillators [3] since it has a wide bandwidth, named Stokes wave, of $40 \mathrm{THz}$ and a maximum at $13 \mathrm{THz}$ from the pump frequency [4]. Moreover, the development of high-power rare-earth doped fiber lasers and diode lasers has attracted the attention of research of Raman amplifiers and Raman fiber laser in recent years $[5,6]$.

On the other hand, an extreme spectral broadening of light in optical fibers is known as supercontinuum generation (SG) and is due to the combination of linear and nonlinear phenomena. SG was first observed in 1970 [7], and like SRS, it has taken advantage of the improvement of high-power laser sources. The extensive study of SG has allowed us to classify some nonlinear phenomena according to two parameters: (i) the dispersion regime of the fiber with respect to the pump laser wavelength, whether is normal or anomalous dispersion regime; and (ii) the temporal regime of the laser, whether it is ultrashort (femtosecond) or short (picosecond, nanosecond) pulse regime $[8,9]$. When pumping in the nor- mal dispersion regime, nonlinear effects such as self-phase modulation (SPM), four-wave mixing (FWM), and SRS have been found responsible for spectral broadening, whereas in anomalous dispersion regime phenomena such as modulation instability (MI) and soliton dynamics dominate the increase of spectral bandwidth [9]. Hence the importance of knowing the properties of both pump source and optical fiber used to understand the dynamics which broadens the pulse.

Among the optical fibers that are most used as a nonlinear medium in SG are photonic crystal fiber [10] because of their enhanced properties, nevertheless, conventional telecom optical fiber has been proven as an efficient medium for SG [11]. Supercontinuum (SC) spectra have found application in the medical field, for example, in optical coherence tomography, where the resolution of an image taken with this technique is the inverse of the spectral bandwidth of the light source $[12,13]$.

In this manuscript, the spectral broadening evolution of a microchip pulsed laser with an operating wavelength of $1064 \mathrm{~nm}$ and temporal width of $700 \mathrm{ps}$ is investigated. Fibers used as a nonlinear medium were high-numerical aperture fiber (HNAF) and non-zero dispersion-shifted fiber (NZDSF). The evolution was studied using input power variation using neutral density filters, allowing a discrete power variation but enough to understand the nonlinear effects involved. Results show the presence of SPM, which initially broadens the pulse, and FWM as well as SRS contribute as symmetric gain bands around the pump. As input power increases, cascaded SRS dominates the broadening up to five Stokes and a total spectral width of $850 \mathrm{~nm}$. 


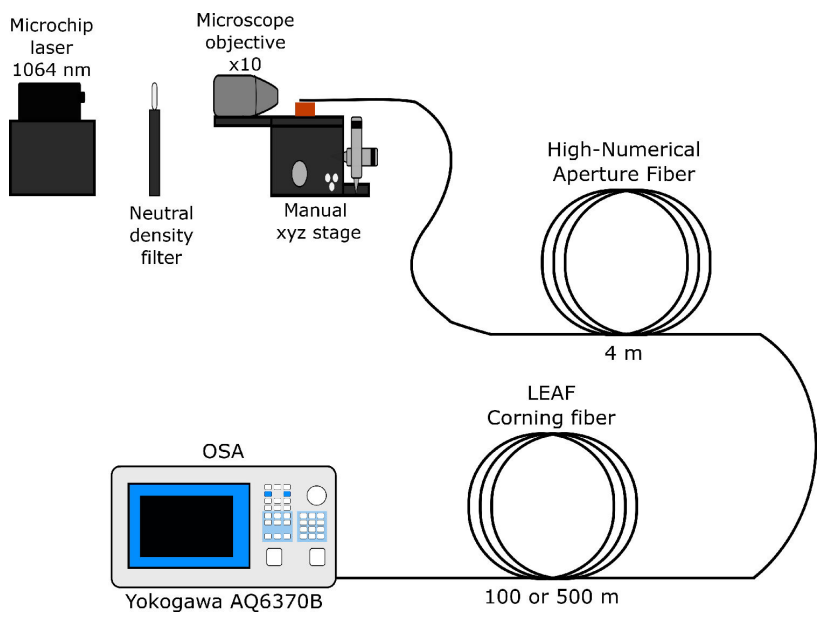

FIGURE 1. Experimental setup for input power variation.

\section{Experimental setup}

The experimental setup is shown in Fig. 1. The pump laser used was a microchip pulsed laser with an operating wavelength of $1064 \mathrm{~nm}$, temporal width of $700 \mathrm{ps}$, a repetition rate of $8.5 \mathrm{kHz}$, an average output power of $22.21 \mathrm{~mW}$, and peak power of $2.442 \mathrm{~kW}$. Neutral density filters with wide bandwidth could be put together to add their optical densities in order to decrease the total transmittance $(\mathrm{T})$, which could be selected in discrete values from 0.01 to 1 and decrease the input power coupled to the fiber. Fibers used were HNAF with a numerical aperture of 0.21 and a LEAF Corning fiber, an NZDSF with zero dispersion around $\lambda_{Z D} \approx 1530 \mathrm{~nm}$. Three lengths were tested in order to study the properties of each fiber and how these are related to the development of present nonlinear phenomena. The lengths were $4 \mathrm{~m}$ of HNAF, later $100 \mathrm{~m}$ of NZDSF were spliced to the short HNAF, and finally, the $100 \mathrm{~m}$ fiber section was cleaved, and a $500 \mathrm{~m}$ NZDSF was then spliced to the HNAF. Numerous combinations of neutral density filters were used in order to change input power from pump laser and observe output spectra through an optical spectrum analyzer (OSA) with a $0.2 \mathrm{~nm}$ resolution.

\section{Results}

\subsection{Experimental results}

First fiber used was the HNAF. This fiber was chosen since it has a smaller effective area than conventional telecom fibers such as SMF-28 or NZDSF. A smaller effective area increases the nonlinearity of the fiber because it is inversely proportional to the nonlinear coefficient $\gamma$. Figure 2 shows the output spectra when filters with a transmittance of $\mathrm{T}=$ $0.1,0.158$, and 0.199 were used. The first filter $(\mathrm{T}=0.1)$ allows enough input power to observe first Stokes with a peak power at $1116.24 \mathrm{~nm}$ and initial width of $18 \mathrm{~nm}$. Moreover, small side lobes around the pump can be observed, which are an experimental evidence identified as FWM [14]. Thus, it is important to point out that nonlinear phenomena happen in a short length of the conventional fiber and with only a $10 \%$ of input pump power coupled. Furthermore, with the filter of transmittance $\mathrm{T}=0.158$ is possible to observe the growth of an Anti-Stokes by Raman effect with a peak power at $1018.24 \mathrm{~nm}$, i.e., a higher frequency shift of $12.7 \mathrm{THz}$ from pump frequency.

Additionally, there is a generation of narrow new frequencies at $\lambda_{1}=966.64 \mathrm{~nm}$ and $\lambda_{S 2}=1184.4 \mathrm{~nm}$, where the latter does not grow as a second Stokes by cascaded SRS; instead, its origin might be an FWM phenomenon between $\lambda_{1}$ and $\lambda_{S 2}$ since both are symmetrically separated by $28 \mathrm{THz}$ from the pump. It is important to point out that these sidebands are described as an FWM process, and not as modulation instability. Both phenomena describe the frequencydomain and time-domain of the same physics $[9,15]$, and we describe the spectral or frequency picture of the supercontinuum evolution. Furthermore, although MI is commonly considered a phenomenon exclusively of the anomalous dispersion regime, MI has been proven to occur both mathematically and experimentally in the normal dispersion regime [10], however, one would need to fulfill phase-matching conditions with a negative higher-order dispersion parameter $\left(\beta_{4}<0\right)$ in the operation wavelength of the pump or phasematched orthogonally polarized electric field components. Nevertheless, we do not expect one of both conditions to be met with the high-numerical aperture fiber since negative high-order dispersion is not common in this type of fiber, and phase-matched orthogonally polarized waves are common in high-birefringence fiber. Besides pump wavelength broadens substantially, which could be due to SPM and cross-phase modulation (XPM) by the interaction between the pump and the present Stokes. Output spectrum with transmittance filter of $\mathrm{T}=0.199$ shows the growth of Anti-Stokes wave by Raman effect as well as the Stokes and Anti-Stokes waves by FWM; however, $\lambda_{S 2}$ line is absorbed by second Stokes of cascaded SRS, since Raman gain is stronger than parametric gain in these conditions.

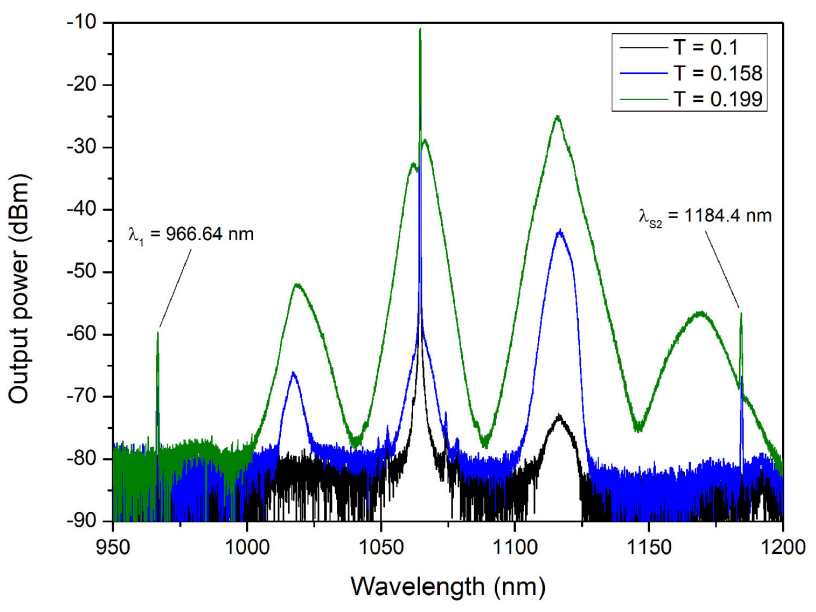

FIGURE 2. Output spectra evolution for low transmittance filters and HNAF. 


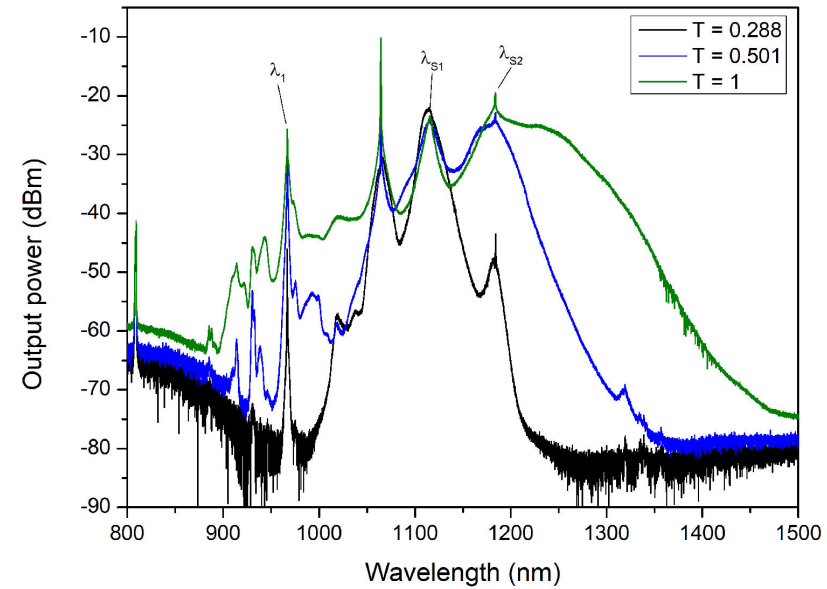

FIGURE 3. Set of measurements for medium transmittance filters and SC spectrum for HNAF.

Figure 3 shows the output spectra for filters with a transmittance of $\mathrm{T}=0.288,0.501$, and no filter used $(\mathrm{T}=1)$. Filter with $\mathrm{T}=0.288$ shows a relevant growth of first and second Raman Stokes as well as increased absorption of Raman Anti-Stokes wave, as expected. However, the output power in the range from 1030 to $1050 \mathrm{~nm}$ does not fall off, in fact, it is relatively flat. Also, there is a power increase and spectral broadening of the $\lambda_{1}$ Anti-Stokes line. This could be due to the interaction of SPM and XPM between the $\lambda_{1}$ and the pump. Spectral broadening continued as a filter with a transmittance of $\mathrm{T}=0.501$ was used, especially for the second Stokes wave and shorter wavelengths than $\lambda_{1}$.

Finally, output spectrum without a filter $(\mathrm{T}=1)$ shows the complete SC achieved, with the first Stokes peak at $\lambda_{S 1}=1115.44 \mathrm{~nm}$, second Stokes at $\lambda_{S 2}=1184.4 \mathrm{~nm}$, respectively, and a total width of $500 \mathrm{~nm}$, from 900 to $1400 \mathrm{~nm}$. The first Stokes have a frequency gain maximum of $12.8 \mathrm{THz}$ away from the pump. Figures 2 and 3 probes that a short length of HNAF ( $4 \mathrm{~m}$ ) is sufficient to observe nonlinear phenomena with our laser pump source.

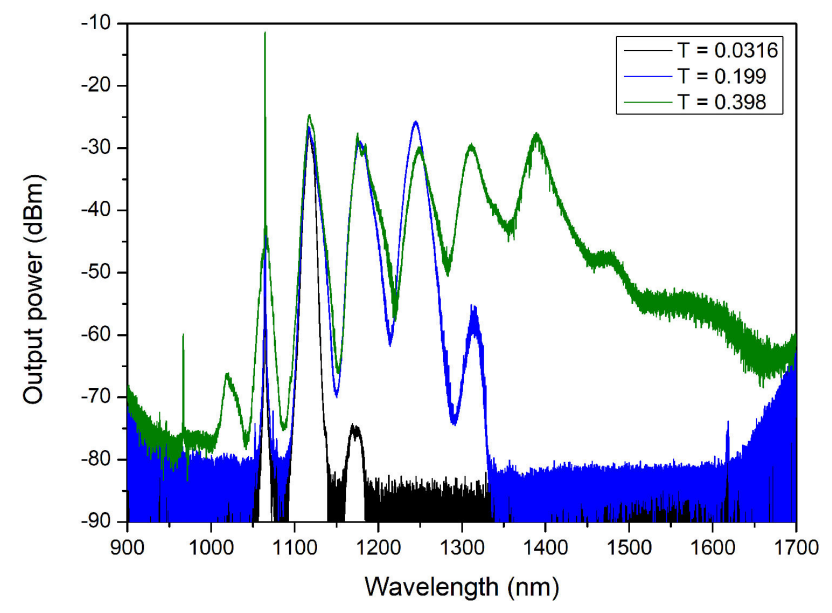

FIGURE 4. Cascaded SRS evolution for $104 \mathrm{~m}$ length fiber.

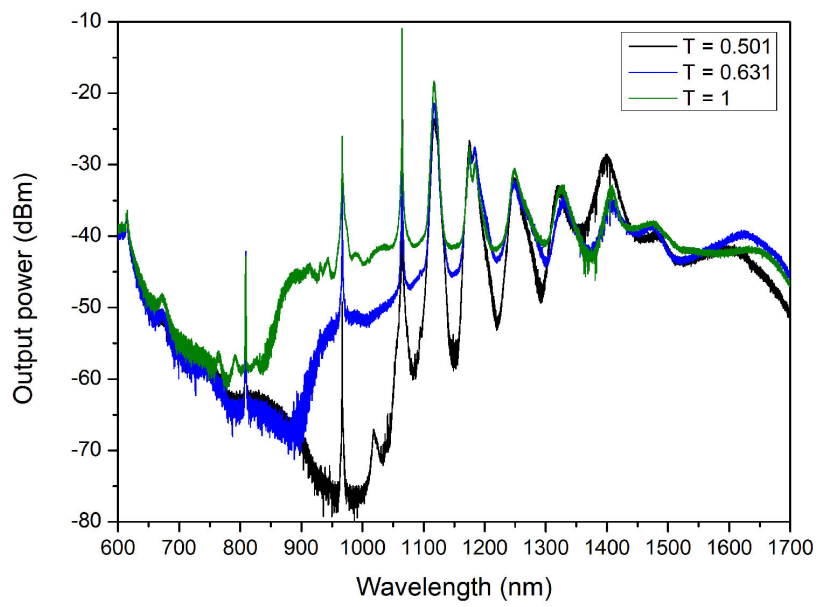

FIGURE 5. SC spectra for $104 \mathrm{~m}$ length fiber.

The next group of measurements was carried out with $100 \mathrm{~m}$ of NZDSF spliced to the HNAF. In Fig. 4, the first filter used had a transmittance of $\mathrm{T}=0.0316$ and enabled the generation of first Raman Stokes and partially a second Stokes. Using a filter of lower transmittance than the previous experiment shows that and increase in fiber length benefits the development of nonlinear effects. Next filter used with a transmittance of 0.199 exhibits the generation of a third and fourth Stokes in the output spectrum. The next, filter with $\mathrm{T}$ $=0.398$ allows enough input power to observe a complete the growth of fourth and fifth Stokes wave, as well as the AntiStokes due to Raman effect and the same Anti-Stokes due to FWM whose origin takes place in the first fiber length, as detailed in the previous experiment. Furthermore, it goes up to $1700 \mathrm{~nm}$, limited by the OSA upper span.

Figure 5 shows the output spectra for filters with $\mathrm{T}=$ 0.501 and 0.631 , as well as without filter. Measurements with filters exhibit a general broadening of output spectrum, especially for wavelengths lower than the pump. With the absorption of the Raman Anti-Stokes, $\lambda_{1}$ line grew, which seems to help increase the broadening for the higher frequency section. Finally, the output spectrum without filter shows a total of

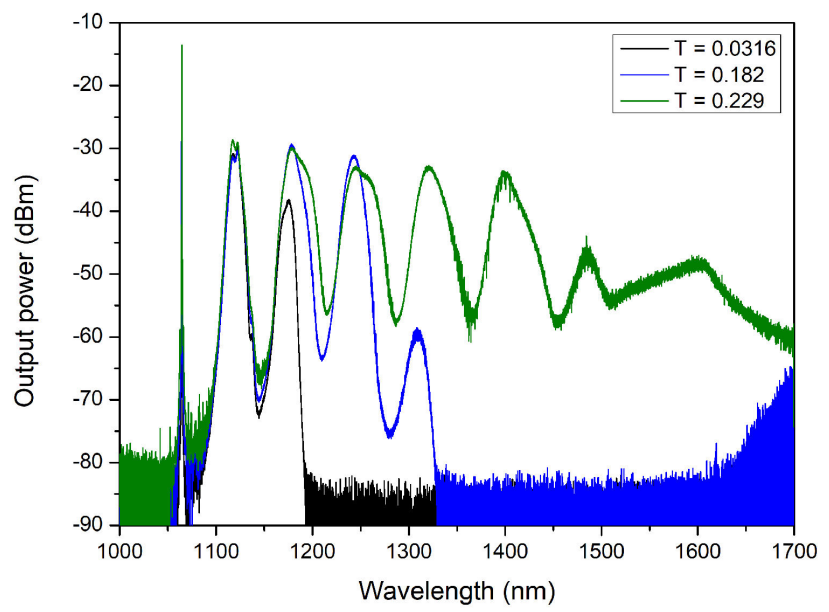

FIGURE 6. Cascaded SRS evolution for $504 \mathrm{~m}$ length fiber. 


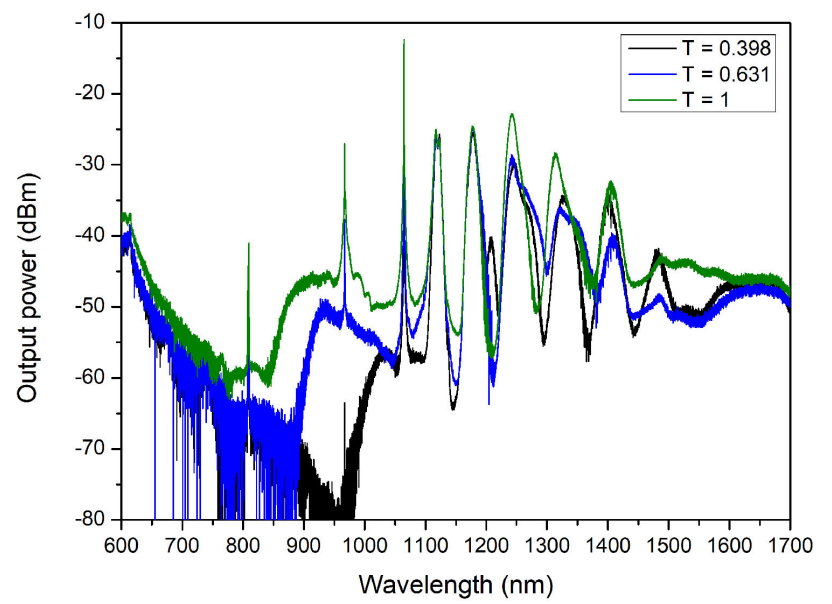

FIGURE 7. SC spectra for medium filter transmittance and $504 \mathrm{~m}$ length fiber.

five Stokes waves, each with a wider gain bandwidth. SC spectrum has a total width of $850 \mathrm{~nm}$, from 850 to $1700 \mathrm{~nm}$, as well as a power variation of $3 \mathrm{~dB}$ in the range from 1500 to $1600 \mathrm{~nm}$, an important spectral band for telecommunications.

The last configuration of this experiment was the HNAF spliced to $500 \mathrm{~m}$ of NZDSF. Figure 6 shows the output spectra using filters with $\mathrm{T}=0.0316,0.182$ and 0.229 . The first filter allowed enough coupled input power for the generation of two Stokes, while with the filter $\mathrm{T}=0.182$ was possible to generate up to a fourth Stokes wave. Next, with the increase of transmittance to 0.229 , an accelerated output spectral broadening happens since a sixth Stokes at $1484.2 \mathrm{~nm}$ appears, and the total spectral width is $636 \mathrm{~nm}$ starting from the pump.

Figure 7 shows the last set of measurements for filters with transmittance $\mathrm{T}=0.398,0.631$, and without a filter. Using the filter with $\mathrm{T}=0.398$ improved the output spectrum flatness for longer wavelengths than sixth Stokes and increased the spectral width for shorter wavelengths than the pump, this time without the appearance of a Raman AntiStokes. The filter with $\mathrm{T}=0.631$ continues allowing the general growth of the spectrum and was it enough input coupled power for the spectral line $\lambda_{1}$, originated in the first few meters of HNAF, to be observed. However, sixth Stokes attenuates. Last, without filter SC, the spectrum shows again a total of five Stokes and a total width of $850 \mathrm{~nm}$, with a power variation of $2.75 \mathrm{~dB}$ in the 1500 to $1600 \mathrm{~nm}$ wavelength range.

\subsection{Numerical results}

The experimental study presented was complemented with a numerical analysis of the spectral evolution as fiber length increased. The simulation was done following the steps and code for pulse propagation in optical fibers, as described by J. C. Travers, M. H. Frosz, and J. M. Dudley in Chapter 3 of Ref. [9], which solves the Generalized Nonlinear Schrödinger Equation (GNLSE) in the frequency domain, and is

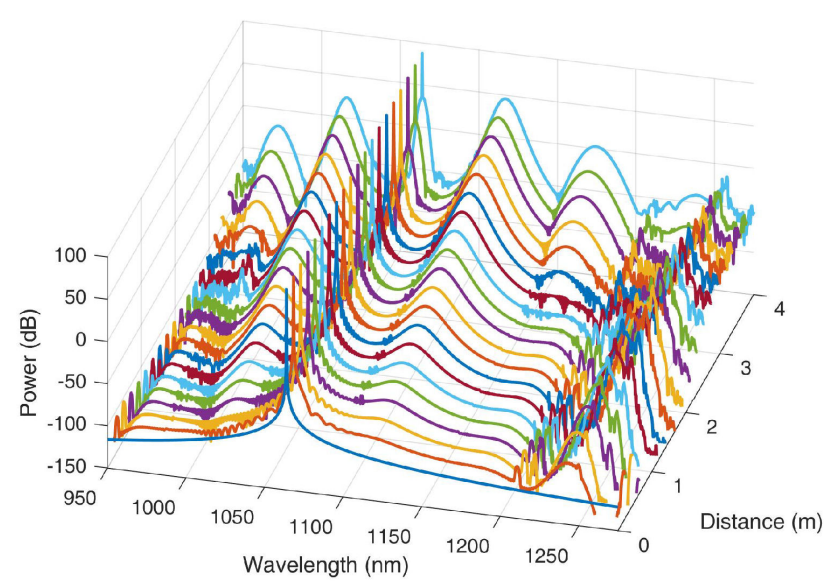

FIGURE 8. Spectral evolution of cascaded SRS and SG by the solution of the GNLSE

$$
\begin{aligned}
\frac{\partial \tilde{A}^{\prime}}{\partial z} & =i \frac{\gamma \omega}{\omega_{0}} e^{-\tilde{L} z} \\
& \times \mathcal{F}\left\{A(z, T) \int_{-\infty}^{\infty} R\left(T^{\prime}\right)\left|A\left(z, T-T^{\prime}\right)\right|^{2} d T^{\prime}\right\}
\end{aligned}
$$

where the frequency dependence of the nonlinear coefficient and the linear operator $\tilde{L}$ is ignored. This change of variable $\tilde{L}$, which includes the dispersion and loss coefficients, makes the GNLSE an ordinary differential equation (ODE) and can be solved easily by using standard ODE codes. Such code was helpful to understand the mechanisms which broaden the pulse to SG. Moreover, parameters measured in this work were input for a $4 \mathrm{~m}$ fiber length, simulating the HNAF used experimentally. Figure 8 shows the spectral evolution for a propagating pulse in a $4 \mathrm{~m}$ length fiber, which is totally dominated by cascaded SRS, with first and second Stokes at 1117 and $1175 \mathrm{~nm}$, respectively. Also, results show the appearance of first and second Raman Anti-Stokes, whose known experimental absorption is not considered in code; however, it was already proved above that it exists.

\section{Discussion}

Results presented in this work showed the SC spectral evolution dominated by cascaded SRS and FWM. Also, SPM has an important role at the start of the broadening, but it is not as relevant as the spectrum extends to longer wavelengths since induced pulse broadening by SPM is inversely proportional to the temporal pulse duration [15]. On the other hand, cascaded SRS stops about the zero-dispersion wavelength (ZDW) of the fiber used; however, the spectrum keeps broadening further from ZDW. This has been explained in other works as a random sequence of ultrashort solitons that experience a self-frequency shift [15], complex parametric phenomena, or soliton components due to the Raman effect [16]. Furthermore, near ZDW, phase-matching conditions could be easily met, hence, parametric gain due to FWM decreases the 
Raman gain coefficient and could be reduced to zero [17]. Besides this parametric gain generates two side lobes (also named Stokes and Anti-Stokes waves) shifted to symmetrical frequencies about the pump frequency, where the shift depends on the wavelength of the pump and how far it is from the ZDW of the fiber [15]. If the pump wavelength is in the normal dispersion regime and is far away from ZDW, side lobes are narrow and far from pump. This is the case of our experiment (Fig. 2, $\mathrm{T}=0.158$ ). As pump wavelength is near ZDW, the side lobes' bandwidth increases and shifts closer to the pump. This has been demonstrated for conventional optical fibers, used in this work, and in microstructured fibers [18], highly nonlinear optical fibers, and dispersion-shifted fibers [19]. Moreover, Fig. 2 shows how lower-frequency narrow side lobe is in the Raman second Stokes gain bandwidth, which could induce to a suppression or enhancement of the Raman Stokes [15]. As shown, by increasing the input pump power, the Raman Stokes is enhanced since its bandwidth increases.

Thus, the use of HNAF improves the spectral broadening for shorter wavelengths since the Anti-Stokes at $966.64 \mathrm{~nm}$ generated by FWM stays for output spectra in longer lengths of NZDSF.

As input power increases, cascaded SRS dominates the broadening in lower frequencies; whereas, a combination of what we believe is XPM and FWM broadens the spectrum for higher frequencies than the pump frequency. With a total fiber length of 104 and $504 \mathrm{~m}$ is possible to see an SC output spectrum with a high flatness in the wavelength range from 1500 to $1600 \mathrm{~nm}$, where single mode fibers have their lowest power loss. This flattening has been described in the literature as the interaction among these low frequencies through XPM and SRS [10]. Moreover, numerical results solving the GNLSE also show a spectral broadening dominated by SRS for a $4 \mathrm{~m}$ fiber length.

\section{Conclusion}

In summary, SG was demonstrated for short and long lengths of conventional optical fibers where cascaded SRS dominated the spectral broadening accompanied by FWM at the beginning of fiber length. The spectral evolution was analyzed by means of neutral density filters to control the input power with discrete transmittance variations. Output spectra showed a total of five Stokes waves for $104 \mathrm{~m}$ and $504 \mathrm{~m}$ of fiber, where each Stokes could be used as a gain medium for an optical signal. Moreover, a high flatness in the telecommunications band for single mode optical fiber was achieved. Conventional fibers used in this experiment proved that care must be taken with input power used for optical signal since even in a common communication fiber such as LEAF, nonlinear phenomena were induced.

\section{Acknowledgements}

The authors would like to thank CONACyT for the Project CB-2016-01-286916, UG for the project grant CIIC 238/2020, and Secretaría de Educación Pública, through PRODEP, with post-doctorate support.

In memoriam of Prof. E. A. Kuzin.
1. C. V. Raman and K. S. Krishnan, A new type of secondary radiation, Nature, 121 (1928) 501, https://doi.org/10. $1038 / 121501 \mathrm{co}$

2. C. V. Raman, A new radiation, Indian J. Phys, 2 (1928) 387.

3. R. H. Stolen, E. P. Ippen, and A. R. Tynes, Raman oscillation in glass waveguide, Appl. Phys. Lett., 20 (1972) 62, https://doi.org/10.1063/1.1654046

4. R. H. Stolen and E. P. Ippen, Raman gain in glass optical waveguides, Appl. Phys. Lett., 22 (1973) 276, https: //doi.org/10.1063/1.1654637

5. S. A. Babin, E. A. Zlobina and S. I. Kablukov, Multimode fiber Raman lasers directly pumped by laser diodes, IEEE J. Sel. Top. Quant., 24 (2018) 1400310, https://doi.org/10. 1109/JSTQE.2017.2764072

6. Y. Chen, T. Yao, L. Huang, H. Xiao, J. Leng and P. Zhou, 2 $k W$ high-efficiency Raman fiber amplifier based on passive fiber with dynamic analysis on beam cleanup and fluctuation, Opt. Express, 28 (2020) 3495, https://doi.org/10.1364/ $\mathrm{OE} .383683$

7. R. R. Alfano and S. L. Shapiro, Emission in the region 4000 to 7000 A via four-photon coupling in glass, Phys.
Rev. Lett., 24 (1970) 584, https://doi.org/10.1103/ PhysRevLett.24.584

8. V. V. Alexander, O. P. Kulkarni, M. Kumar, C. Xia, M. N. Islam, F. L. Terry, M. J. Welsh, K. Ke, M. J. Freeman, M. Neelakandan and A. Chan, Modulation instability initiated high power allfiber supercontinuum lasers and their applications, Opt. Fiber. Technol., 18 (2012) 349, https://doi.org/10.1016/ j.yofte.2012.07.014

9. J. Travers, M. Frosz, and J. Dudley, Nonlinear fibre optics overview. In J. Dudley and J. Taylor (Eds.), Supercontinuum Generation in Optical Fibers. (Cambridge: Cambridge University Press, 2010). pp. 32-51, doi 10.1017/ CB09780511750465.004

10. S. Coen, A. H. L. Chau, R. Leonhardt, J. D. Harvey, J. C. Knight, W. J. Wadsworth and P. S. J. Russell, Supercontinuum generation by stimulated Raman scattering and parametric four-wave mixing in photonic crystal fibers, J. Opt. Soc. Am. B., 19 (2002) 753, https://doi.org/10.1364/ JOSAB.19.000753

11. J. C. Hernandez-Garcia, J. M. Estudillo-Ayala, O. Pottiez, R. Rojas-Laguna, R. I. Mata-Chavez and A. Gonzalez-Garcia, Generation of a spectrum with high flatness and high band- 
width in a short length of telecom fiber using microchip laser, Opt. Commun., 292 (2013) 126, https://doi.org/10. $1016 / j . o p t c o m .2012 .11 .076$

12. I. Hartl, X. D. Li, C. Chudoba, R. K. Ghanta, T. H. Lo, J. G. Fujimoto, J. K. Ranka and R. S. Windeler, Ultrahigh-resolution optical coherence tomography using continuum generation in an air-silica microstructure optical fiber, Opt. Lett., 26 (2001) 608,https://doi.org/10.1364/OL.26.000608

13. C. L. Pan, A. Zaytse, Y. J. You and C. H. Li, Fiber-lasergenerated noise-like pulses and their applications. In Fiber Laser, M. C. Paul, Ed. (IntechOpen, 2016). pp. 211-243, http://dx.doi.org/10.5772/61856

14. P. Baldeck and R. Alfano, Intensity effects on the stimulated four photon spectra generated by picosecond pulses in optical fibers, J. Lightwave Technol., 5 (1987) 1712, https: //doi.org/10.1109/JLT.1987.1075465

15. J. M. Dudley, G. Genty and S. Coen, Supercontinuum generation in photonic crystal fiber, Rev. Mod.
Phys., 78 (2006) 1135, https://doi.org/10.1103/ RevModPhys.78.1135

16. U. Tegin and B. Ortac, Cascaded Raman scattering based high power octave-spanning supercontinuum generation in gradedindex multimode fibers, Sci. Rep., 8 (2018) 12470, https: //doi.org/10.1038/s41598-018-30252-9

17. E. Golovchenko, P. Mamyshev, A. Pilipetskii and E. Dianov, Mutual influence of the parametric effects and stimulated $R a$ man scattering in optical fibers, IEEE J. Quantum Electron., 26 (1990) 1815, https://doi.org/10.1109/3.60906

18. L . Zhang, T. H. Tuan, H. Kawamura, T. Suzuki and Y. Ohishi, Optical parametric oscillator based on degenerate four-wave mixing in suspended core tellurite microstructured optical fiber, Opt. Express, 23 (2015) 56299, https://doi.org/10. $1364 / \mathrm{OE} .23 .026299$

19. M. Marhic, K. Y. Wong and L. Kazovsky, Wide-band tuning of the gain spectra of one-pump fiber optical parametric amplifiers, IEEE J. Sel. Top. Quant., 10 (2004) 1133, https: //doi.org/10.1109/JSTQE.2004.835298 